\section{So einzigartig wie meine Praxis}

Bei jedem Smartphone oder Personal Computer ist es längst eine Selbstverständlichkeit, mit CGM Z1.PRO jetzt auch in der Zahnarztsoftware: ein Höchstmaß an Einzigartigkeit und Individualität auf dem Bildschirmhintergrund. Die im Erscheinungsbild einer Praxis angestrebte Corporate Identity erobert die Oberfläche der Zahnarztsoftware, mit dem Ergebnis, dass jeder Mitarbeiter sich im wahrsten Sinne des Wortes in seinem eigenem Aufgabenbereich „wiedersieht“. Z1.PRO bindet nicht nur Logo und Praxisfarben in seine Oberfläche ein, sondern es verbindet diese Vorteile mit einem durchdachten Ausbau an Anwendungsvorteilen. Ein modernes Design steht hier für Übersichtlichkeit und Zeitersparnis.

Seit nunmehr 25Jahren, von Anfang an, ist unsere Praxis mit Zahnarztsoftware-Programmen vertraut. Waren zunächst die Abrechnung und Leistungserfassung die ursprünglichen Königsdisziplinen des 1 . Systems, so begleitet CGM Z1 als multifunktionales Zahnarzt-Informationsinstrument seit 1999 unseren Praxisalltag in der Bewältigung vielfältiger Aufgaben, von A wie Archivierung bis $\mathrm{Z}$ wie Zahlungserfassung. Da verwundert es nicht, dass unser Team aufgrund der neuen Optik neugierig auf die neue Z1.PROOberfläche war und wir zu den ersten Anwenderpraxen gehörten ( $\bullet$ Abb. 1).

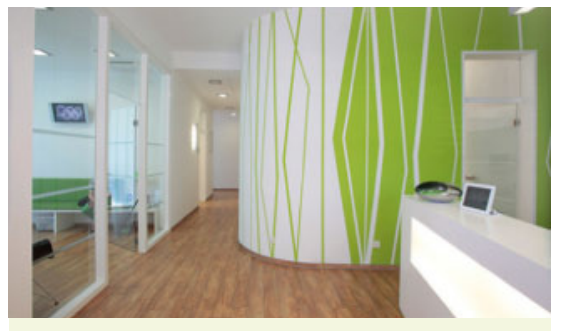

Abb. 1 Die Praxis Dr. Hermeler.

\section{Einzigartig auf den 1. Blick}

Ein Blick genügt, der Startbildschirm ist nicht mehr identisch mit der Oberfläche wie bei tausenden anderen Systembenutzern, nein, er ist jetzt in 5 wichtigen Bereichen einmalig auf unsere Praxis und unser Team ausgelegt ( $\bullet$ Abb.2). Erstens erscheint mittig oben auf allen Arbeitsplätzen das Bild unserer Mannschaft im Bannerformat (Punkt 1). Hier kann individuell

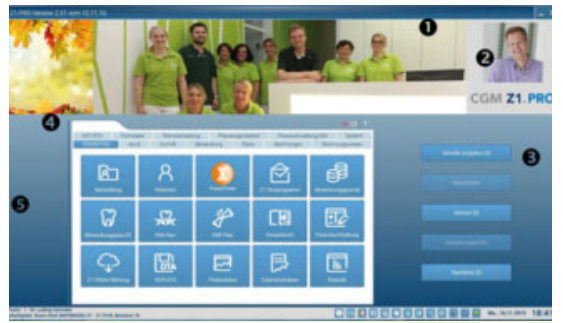

Abb. 2 Z1.PRO, Hauptmenü am Arbeitsplatz Chefbüro. das hinterlegt werden, was die Praxis ausmacht, bei uns steht nicht nur hier das Team im Vordergrund. Rechts davon sieht man den eingewählten Behandler oder Mitarbeiter (Punkt 2). Das ist z.B. in der Prophylaxe hilfreich, wo mehrere Fachkräfte agieren und wo über ein Zahlenkürzel hinaus die korrekte Zuordnung im Bild zu eingetragenen Leistungen und zu den Patienten in der weiteren Betreuung erleichtert wird. Auch sehen sich alle Teammitglieder im wahrsten Sinne im Programm wieder. Gleiche Vorteile liegen in der Mehrbehandler-Praxis auf der Hand bzw. sind augenscheinlich.

Der 3. Punkt gibt das individuelle Mitarbeiterprofil wieder, das direkt unter dem Profilbild mit der Warteliste, ungelesenen Nachrichten, Notizmöglichkeiten und anstehenden Aufgaben aufwartet. Veränderungen machen Spaß, wenn z. B. praxisinterne Nachrichten jetzt mit dem Bild des Verfassers erfolgen. An 4. Stelle steht der Favoriten-Reiter neben den anderen Software-Bausteinen in der Mitte des Startbildschirms. Je nach Arbeitsplatz stehen bestimmte Bestandteile des Zahnarztinformations-Systems im Vordergrund. Über Favoriten hat jedes Teammitglied von der Abrechnungsmitarbeiterin über die Assistenz bis zu den Behandlern alle Funktionen im Direktzugriff, die sie oder er am häufigsten nutzt. Die Favoriten lassen sich nach Mitarbeiter oder Arbeitsplatz individuell auswählen, wodurch viel Zeit bei den täglichen Routineabläufen eingespart wird. Wird doch einmal ein Programmteil gesucht, der selten genutzt wird, kann er einfach und schnell unter dem neuen Reiter „Suchen“ unter Eingabe von 3 Buchstaben gefunden werden. Mein 5. Punkt ist die farbliche Grundgestaltung, die über die Bildschirmdarstellung in Farbton, Sättigung und Helligkeit ganz nach Geschmack und Vorliebe an jedem Rechner gewählt werden kann. Der Rezeptionscomputer hat ähnlich der Farbge- staltung im Empfangsbereich unserer Praxis einen frischen Grün-Gelb-Ton ( Abb. 3), im Röntgenraum erscheint ein dunklerer Blau-Ton, an meinem Bürorechner mag ich die hellblaue Variante. Corporate Identity setzt sich auf diese Weise sinnvoll auch im optischen Erscheinungsbild des Computersystems fort.

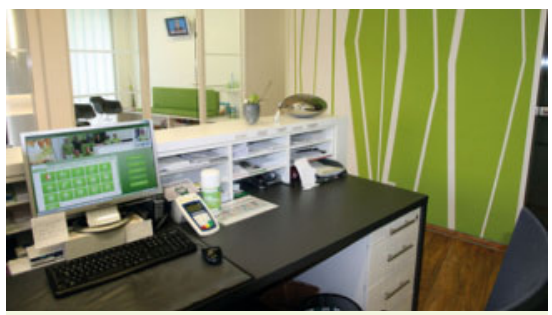

Abb. 3 Der Rezeptionsrechner im Eingangsbereich, die Software ist hier farblich an die Praxisräume angepasst.

Wesentlich zum besseren Überblick bei den Programm-Dialogen trägt ein neues Feature, die Z1.PRO Skalierbarkeit, bei. Das Design der Software passt sich der Oberfläche des jeweiligen Endgeräts an. Vom Anwender eingestellte Größen und Positionen im Dialog des Systems werden abgespeichert ( $\bullet$ Abb.4).

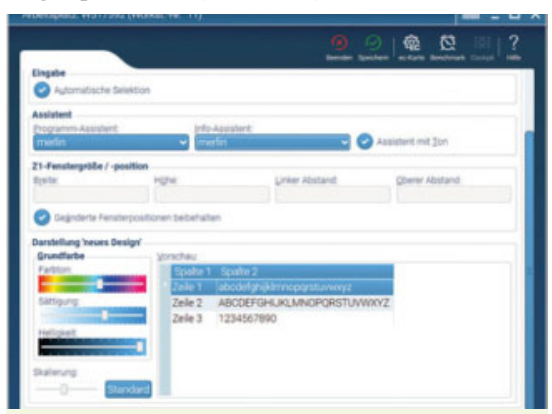

Abb. 4 Die Z1.PRO-Skalierbarkeit.

Der technische Umstieg von CGM Z1 auf CGM Z1.PRO gestaltet sich per CD völlig unkompliziert, einfach und sicher durch die automatische Datenübernahme. Hierzu wird kein Technikereinsatz benötigt. In unserer Praxis war kein Hardware-Wechsel notwendig.

\section{Zeitsparend und wirtschaftlich \\ $\nabla$}

Das Höchstmaß an Individualisierung und an zeitsparenden Konfigurationsmöglichkeiten kann im Praxisalltag nur erfolgreich zum Tragen kommen, wenn es in einem System eingebunden ist, das alle Praxisaufgaben bewältigen kann. Hier ist die modulare Z1-Vielfältigkeit die Basis für die neue Generation CGM Z1.PRO im übersichtlichen, intuitiv einfachen und schnellen Arbeiten am Computer. Alle 


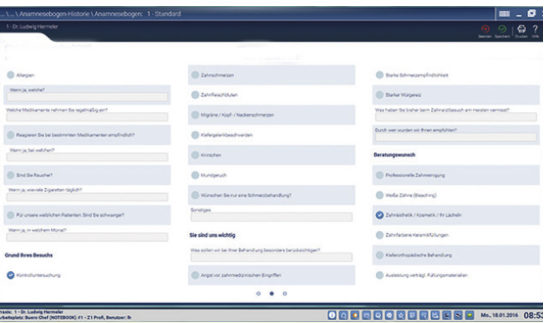

Abb. 5 Der Z1-Anamnesebogen.

Programmteile sind intelligent verknüpft und bringen gewünschte Informationen mit wenigen Klicks zutage. Das fängt mit dem Z1-Anamnesebogen an, der alle vom Patienten angegebenen Informationen automatisch in die Kartei einfließen lässt ( $\triangle$ Abb. 5), sodass sie bei der Therapieplanung berücksichtigt werden können, z. B. erscheint dann der Beratungswunsch „Zahnästhetik“ im Z1-Premium Behandlungsmanager ( $\bullet$ Abb. 6 ).

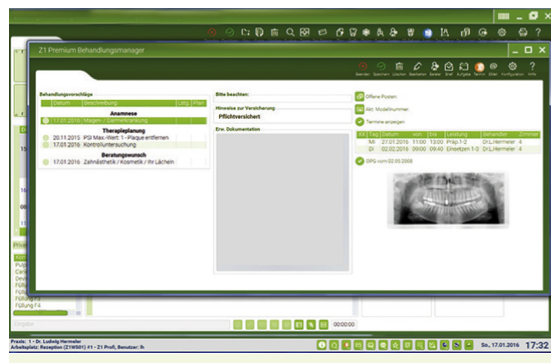

Abb. 6 Der Z1-Premium Behandlungsmanager.

Dieses Modul übernimmt zeitsparend und übersichtlich alle Patientenwünsche, Risiken, Therapievorschläge und Versicherungsinformationen zur Patientenberatung. Die Leistungseingabe kann über die Komplex-und Dokumentations-Erfassung schnell und intuitiv erfolgen. Damit ist eine vollständige Dokumentation sicher und zeitsparend möglich, zusätzliche Notizen sind jederzeit zügig erfasst. Mit einem Klick zeigt der Z1-Patientenstatus übersichtlich und komprimiert Informationen über die Besuche in der Praxis, die Recall-Daten, die geplanten ZE-Anträge, die Zahlungsmoral und die Umsätze für die jeweilige Person an ( 0 Abb. 7). Der Behandler hat somit alles Wesentliche im Blick. Durch die sehr gute Vernetzung von Z1.PRO mit dem CGM PRAXISTIMER PRO können alle Termine des Patienten in der Karteikarte oder über die Tagesübersicht im Timer eingesehen werden ( $\mathbf{O}$ Abb. 8)

Die wirkungsvolle Gegenüberstellung von Regel- und Alternativ-Versorgungen im Z1-Patientenberater - einfach aus der

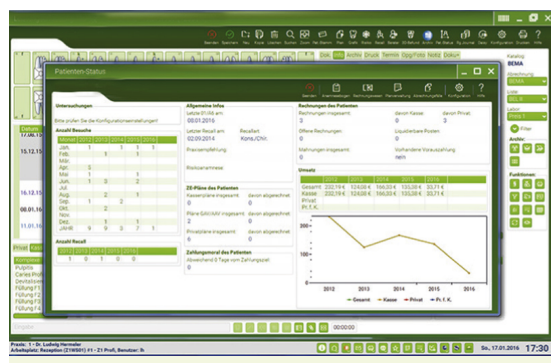

Abb. 7 Der Z1-Patientenstatus.

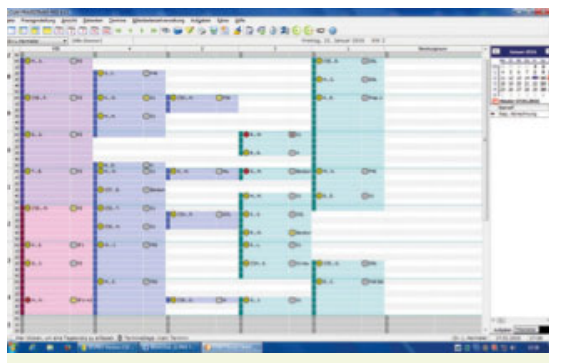

Abb. 8 Patiententermine im CGM PRAXISTIMER PRO.

Zahnersatzplanung heraus - ist ideal für die Patientenaufklärung (๑ Abb.9).

In dem vom TÜV zertifizierten CGM Praxisarchiv werden alle Dokumente des Patienten (nicht nur Röntgen-, extra- und intraorale Bilder) der richtigen Karteikarte zugeordnet und sind in Sekundenschnelle auffindbar. Die Darstellung des Archivs am Monitor des Behandlungsstuhls (๑ Abb. 10) unterstützt den Behandler und ist für den Patienten ein sichtbares Zeichen des digitalen Fortschritts der Praxis. Alle Vorgaben des neuen Patienten-

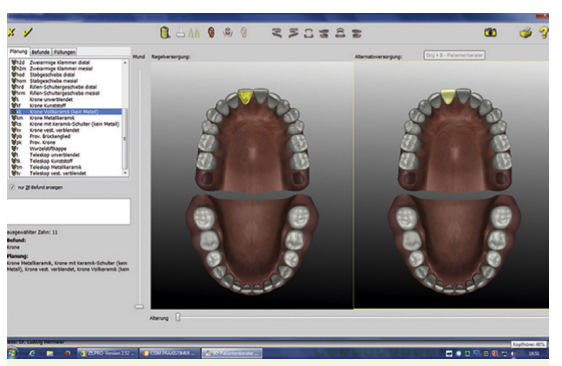

Abb. 9 Der Z1-Patientenberater mit gegenübergestellten Versorgungsalternativen.

rechtegesetzes bezüglich einer überprüfbaren, rechtssicheren Archivierung der Karteikarten werden mit dem Modul Z1Karteikartenarchivierung erfüllt. Die entsprechenden Z1.PRO Module im Bereich Praxiscontrolling sorgen dafür, dass das Abrechnungs-und Budget-Wesen kein Buch mit 7 Siegeln ist, das nur von der KZV oder den Krankenkassen geöffnet wird. Anhand der Z1 Module Z1 Praxisstatus Plus, Z1-1-Klick-Status, Z1-100-Fall-Statistik und Z1 Honorarverteilungsmaßstab

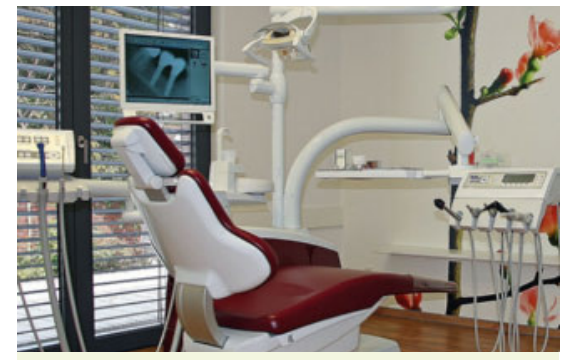

Abb. 10 Das CGM Praxisarchiv ist direkt am Behandlungsstuhl abrufbar.

kann der Behandler auf Basis der bisherigen Leistungen Abrechnungspotenziale und Differenzen zu Budgetgrenzen in übersichtlicher Darstellung erkennen. Ferner kann er Prognosen anhand der in der Software eingetragen Werte erstellen und gegebenenfalls einen Schritt früher reagieren, z.B. in der Kontrolle der Dokumentation in „abweichenden“ Werten, bevor mögliche Nachfragen diesbezüglich seitens der KZV zeitversetzt eintreten.Damit sind die individuellen Möglichkeiten der Z1 Praxisunterstützung bei weitem nicht erschöpft. Beispielhaft seien die Module CGM PRAXISTIMER Pro \& CGM LIFE eSERVICES (digitale Terminvergabe) und die Z1-Spracherkennung („freie Hände“ z.B. bei der PA Befundeingabe) erwähnt. Jede Praxis kann individuell nach ihren Bedürfnissen eine optimale Gesamtlösung aus einem breiten Spektrum an Einzelmodulen zusammenstellen.

\section{Fazit \\ $\nabla$}

Die Z1.PRO Oberfläche ist mehr als nur eine „oberflächliche“ Auffrischung des bewährten Z1 Marktführers. Die neue CGM Generation Z1.PRO setzt erneut den Maßstab für Übersichtlichkeit, Individualisierung, Zeitersparnis, Schnelligkeit und Aufbereitung wirtschaftlicher Erfolgsparameter. Die Individualität unserer Praxis spiegelt sich in den gestalterischen Möglichkeiten in unserem Zahnarzt-Software-Programm wieder, optisch und inhaltlich. Um es auf einen Nenner zu bringen: Unser Team möchte auf CGM Z1.PRO einfach nicht mehr verzichten.

\section{Interessenkonflikt}

Der Autor benutzt seit 1991 käuflich erworbene Software von CGM und seit Oktober 2015 CGM Z1.PRO.

\section{Korrespondenzadresse}

Zahnärzte Mesum

Dr. Hermeler \& Kollegen

Rheiner Straße 30

48432 Rheine-Mesum

info@zahnarzt-mesum.de 